\title{
OPTICAL STUDY OF AN ASYMMETRIC DOUBLE QUANTUM WELL SYSTEM: CdTe/CdMnTe
}

\author{
I. Lawrence, G. Feuillet, H. Tuffigo, C. Bodin, J. Cibert \\ CEA-CNRS II-VI Research Group, B.P. 85, 38042 Grenoble, France
}

AND W.W. RÜHLE

Max-Planck-Institut für Festkörperforschung

Heisenbergstr. 1, W 7000 Stuttgart 80, Germany

\begin{abstract}
Carrier tunneling through CdMnTe barriers of different thicknesses is investigated in $\mathrm{CdTe} / \mathrm{CdMnTe}$ asymmetric double quantum wells. Steady-state photoluminescence at $1.8 \mathrm{~K}$ and time-resolved photoluminescence experiments between $10 \mathrm{~K}$ and $50 \mathrm{~K}$ were performed.
\end{abstract}

PACS numbers: 78.66.Hf; 78.20.Ls, 78.47.+p

Semimagnetic semiconductors are of great interest because of their novel properties [1]. In particular, the strong interaction between the spins of carriers and the magnetic ions leads to giant magnetooptical effects such as extremely large Zeeman splittings.

In contrast to previous studies of Mn-based asymmetric double quantum wells (ADQWs) where the quantum wells (QW) were made of CdTe $[2,3]$, in our system the wide well (WW) contains Mn, so as to increase drastically the influence of a magnetic field on the confined levels [4].

In this report we will present steady-state and time-resolved experiments at zero tesla, focusing on the influence of the tunnel barrier thickness on the tunneling.

The samples were grown by molecular beam epitaxy in the (100) direction. The structures were deposited on a CdMnTe buffer layer of the same Mn concentration as the barriers. Table compiles for the samples the Mn concentrations (of the barriers $\left(x_{\mathrm{B}}\right)$, of the WW $\left(x_{\mathrm{W}}\right)$ ), and the thicknesses measured by Reflection IIigh Energy Electron Diffraction (RHEED) oscillations (WW $\left(L_{\mathrm{W}}\right)$, narrow well $\left(L_{\mathrm{N}}\right)$, tunnel barrier $\left.\left(L_{\mathrm{B}}\right)\right)$.

The photoluminescence (PL) spectra are depicted in Fig. 1. The emission of the fundamental excitons of both QWs is observeu: $e_{N}^{1} h h_{N}^{1}$ at lower energy and $e_{\mathrm{W}}^{1} \mathrm{hh}_{\mathrm{W}}^{1}$ at higher energy. The labels $\mathrm{hh}_{\mathrm{N}}^{1}\left(\mathrm{hh}_{\mathrm{W}}^{1}\right)$ mean the first heavy hole level in the narrow CdTe (wide CdMnTe) QW. Note that because the gap increases with the $\mathrm{Mn}$ content, the WW luminescence appears at higher energy. The relative intensity of their luminescence $\left(R=I_{\mathrm{W}} / I_{\mathrm{N}}\right)$ reflects the strength of the coupling. The observed increase in $R$ when $L_{\mathrm{B}}$ increases indicates a less efficient tunneling 
TABLE

\begin{tabular}{l|c|c|c|c|c}
\hline \hline & $\begin{array}{c}x_{\mathrm{B}} \\
{[\% \mathrm{Mn}]}\end{array}$ & $\begin{array}{c}x_{\mathrm{W}} \\
{[\% \mathrm{Mn}]}\end{array}$ & $\begin{array}{c}L_{\mathrm{W}} \\
{[\AA]}\end{array}$ & $\begin{array}{c}L_{\mathrm{B}} \\
{[\AA]}\end{array}$ & $\begin{array}{c}L_{\mathrm{N}} \\
{[\AA]}\end{array}$ \\
\hline $\mathrm{M} 256$ & 11.6 & 3.7 & 143 & 48.7 & 54 \\
$\mathrm{M} 257$ & 11.4 & 3.5 & 145 & 79.5 & 53.6 \\
$\mathrm{M} 258$ & 10.6 & 3.4 & 147 & 113 & 51
\end{tabular}

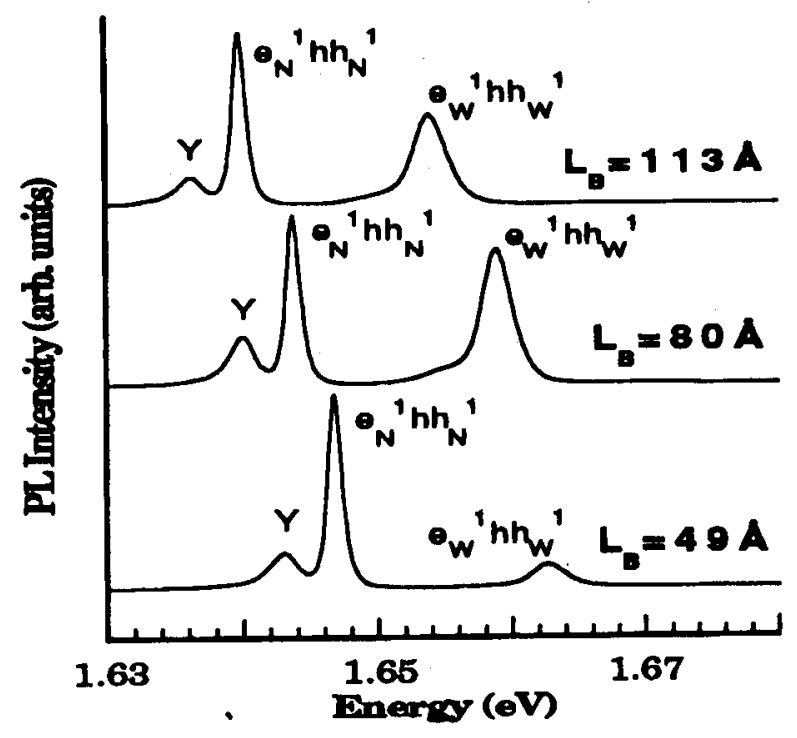

Fig. 1. PL spectra of the three ADQWs having various tunnel barrier thicknesses $(1.8 \mathrm{~K})$. Beside intrinsic excitons $\left(\mathrm{e}_{\mathrm{N}}^{1} \mathrm{~h} \mathrm{~h}_{\mathrm{N}}^{1}, \mathrm{e}_{\mathrm{W}}^{1} \mathrm{~h} \mathrm{~h}_{\mathrm{W}}^{1}\right)$, the lines labeled $Y$ are attributed to localized excitons.

for thicker barriers. However, the sample with $L_{\mathrm{B}}=110 \AA$ does not follow this trend, as it will be confirmed by time-resolved photoluminescence (TRPL).

Figure 2 shows TRPL data of the $L_{\mathrm{B}}=80 \AA \mathrm{ADQW}$ at $50 \mathrm{~K}$. Spectra (a) and (c) are the luminescence decays of the excitonic ground state in CdTe and CdMnTe QWs, respectively, with an excitation above both QWs. The CdMnTe QW luminescence (c) shows an initial fast decay time of $185 \mathrm{ps}$ which is attributed to the tunneling of the excitons from the CdMnTe QW to the CdTe QW. Then, $400 \mathrm{ps}$ after the laser pulse, the luminescence of both CdTe and CdMnTe QWs decreases with a common decay time of $585 \mathrm{ps}$ ((a) and (c)) indicating that at this lattice temperature, a thermal equilibrium is reached in the occupation of the WW and NW excitonic states.

We now compare the luminescence decay of the NW $(\mathrm{CdTe})$ at two different 


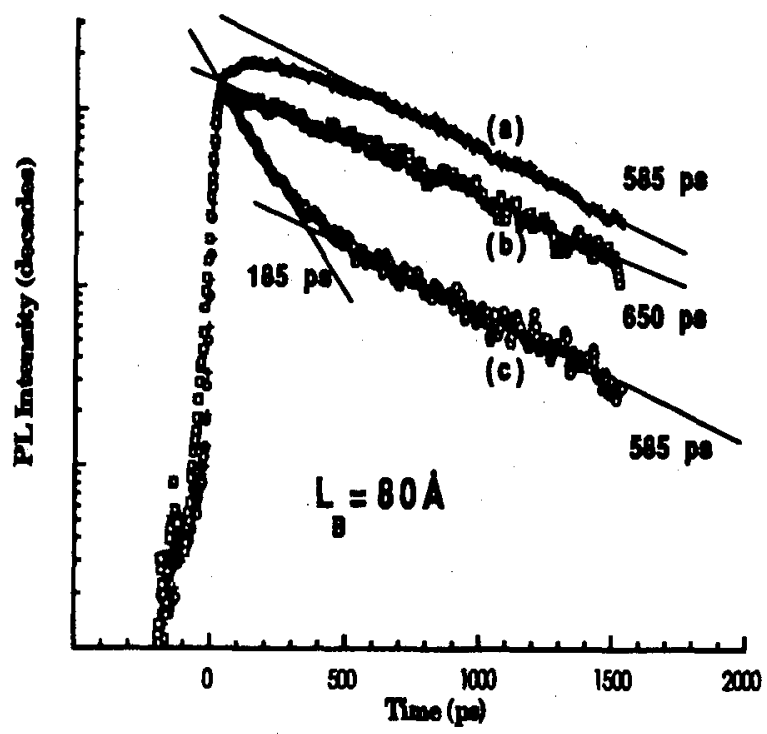

Fig. 2. Evidence of the tunnel effect at $50 \mathrm{~K}$ in the $80 \AA$ thick tunnel barrier sample. (a) and (b) are the luminescence decays of the low energy $Q W(\mathrm{CdTe}$ ) when exciting (a) above or (b) under the high energy QW (CdMnTe). (c) is the luminescence decay of the high energy $\mathrm{QW}$.

excitation energies, first exciting both QWs (a) and second, exciting only the CdTe QW (b): (b) shows a monoexponential luminescence decay starting just after the laser pulse, while (a) presents a decay which starts after a delay of $200 \mathrm{ps}$. This delay must be a result of the arrival of carriers by tunneling from the high energy QW (CdMnTe). This intepretation is confirmed by the fact that this delay is of the same order of magnitude as the luminescence decay time of the high energy QW. We have chosen a sample temperature of $50 \mathrm{~K}$ since cooling of excitons (which can also lead to an initial increase in the luminescence) to this temperature is faster than our time resolution. The measured luminescence decay time $(\tau)$ of the high energy QW depends on the exciton recombination time $\left(\tau_{\mathrm{R}}\right)$ and on the tunneling time $\left(\tau_{\mathrm{T}}\right)$ according to the relation $1 / \tau=1 / \tau_{\mathrm{R}}+1 / \tau_{\mathrm{T}}$. We can deduce tunneling times if we take as the exciton recombination time the characteristic decay time at long times: we get $\tau_{\mathrm{T}}=40 \mathrm{ps}$ for $L_{\mathrm{B}}=50 \AA$ and $\tau_{\mathrm{T}}=270 \mathrm{ps}$ for $L_{\mathrm{B}}=80 \AA$ as well as $110 \AA$. Surprisingly, the samples with $L_{\mathrm{B}}=80 \AA, 110 \AA$ have very similar tunneling characteristics (to be seen also in $\mathrm{PL}$ ). This may be due to nearly resonant tunneling conditions, the ADQW with $L_{\mathrm{B}}=110 \AA$ being closer to the resonance $\left(\mathrm{e}_{\mathrm{W}}^{1} \mathrm{hh}_{\mathrm{W}}^{1}\right.$ with $\left.\mathrm{e}_{\mathrm{W}}^{1} \mathrm{hh}_{\mathrm{N}}^{1}\right)$ [5].

In conclusion, we observe tunneling in ADQWs where the high energy $\mathrm{QW}$ contains Mn. The effect of the tunnel barrier thickness is seen in steady-state PL. Moreover, TRPL experiments evidence tunneling of carriers in the luminescence of both QWs: the departure of the carriers from the CdMnTe QW and their arrival in the CdTe QW is observed. 


\section{References}

[1] J.K. Furdyna, J. Appl. Phys. 64; R29 (1988).

[2] W. Heimbrodt, O. Göede, Th. Köpp, K. Hieke, H.E. Gumlich, Th. Pier, B. Lunn, T. Gregory, J. Crystal Growth 117, 859 (1992).

[3] O. Goede, W. Heimbrodt, K. Hieke, H.E. Gumlich, Th. Pier, B. Lunn, D.E. Ashenford, S. Jackson, J.E. Nicholls, Superlattices Microstruct. 42, 363 (1992).

[4] I. Lawrence, G. Feuillet, H. Tuffigo, C. Bodin, J. Cibert, P. Peyla, A. Wasiela, Superlattices Microstr. 42, 119 (1992).

[5] S. Maacke, N.T. Pelekanos, H. Mariette, M. Zigone, A.P. Heberle, W.W. Rühle, to be published in Physical Review B rapid communications (June 1993). 